\title{
Trace Gas Sensing Based on Laser Photoacoustic Spectroscopy
}

$\mathrm{Ma} \mathbf{Y}^{*}$

National Key Laboratory of Science and Technology on Tunable Laser, Harbin Institute of Technology, Harbin 150001, China

*Corresponding author: Ma Y, National Key Laboratory of Science and Technology on Tunable Laser, Harbin Institute of Technology, Harbin 150001, China, Tel: +8645186412; E-mail: mayufei926@163.com

Received date: November 11, 2016; Accepted date: December 21, 2016; Published date: December 28, 2016

Copyright: (c) $2017 \mathrm{Ma} \mathrm{Y}$. This is an open-access article distributed under the terms of the Creative Commons Attribution License, which permits unrestricted use, distribution, and reproduction in any medium, provided the original author and source are credited.

\section{Introduction}

There is significant meaning for the development of trace gas detection in the atmospheric environment monitoring, long distance detection of explosives and the physiological state of biont. For example, the trace gas of atmosphere, however, always exists with the concentration of pptv-ppmv. Although the concentration is very low, it has a great impact on the environment. Detecting this trace gases' component, concentration and its variation in the interspaces and period precisely have an extraordinary meaning, especially in the basic research fields and applied technique domain.

In the trace gas detection research, there are several effective methods, and it can be divided into two fields, namely spectroscopy and non-spectroscopy. Non-spectroscopy includes gas chromatography, mass spectrometry, semiconductor transducing and electrochemical transducing. The spectroscopy includes Tunable Diode Laser Absorption Spectroscopy (TDLAS), Photo Acoustic Spectroscopy (PAS), Faraday Rotation Spectrum (FRS) and optical fiber sensors. Spectroscopy has the advantages of selectivity and identification. So, the development of spectroscopy technique in the trace gas detection fields has a broad prospect. And it has become a pop research region.

\section{The Fundament of Photoacoustic Spectroscopy}

PAS is an indirect absorption spectroscopy based on photoacoustic effect. The theory framework of photoacoustic effect is shown in Figure 1. When light exciting gas, gas molecules would absorb the radiation and then are stimulated up to high energy level.

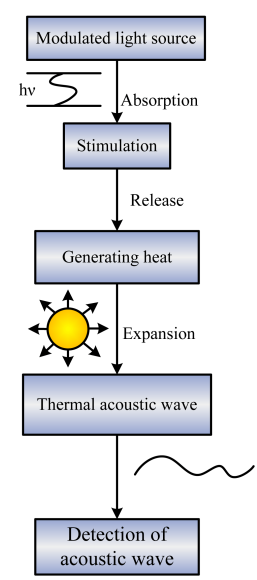

Figure 1: The theory framework of photoacoustic effect.
The stimulating molecules jump to ground state without radiation after inter-collision, then converts light energy into thermal energy. Thus the gas' temperature of local field would change, which lead to gas pressure changing. This process comes into being dilatational wave. If light wave is adjusted periodically, the gas temperature and the pressure will change periodically. Because the frequency of dilatational wave and the adjusted light are equal, and the frequency is within audio frequency field, the dilatational wave would be the acoustic wave. Therefore, we can inverse the gas concentration by detecting the amplitude of acoustic wave signal.

\section{The Fundament of Quartz-Enhanced Photoacoustic Spectroscopy}

Quartz-enhanced photoacoustic spectroscopy (QEPAS), a novel spectrum detection technique, which was firstly reported in 2002 [1]. Compared with TDLAS and PAS, the detection component is replaced by low-cost quartz tuning fork (QTF) in QEPAS. QTF has a stable structure and high Q-factor [2,3]. Besides, it's symmetrical dipole figure lead to an excellent immunity on noise condition [4]. Above these advantages, QEPAS has outstanding features and therefore this research has aroused great attention by many scientists [5].

The configuration of detection system with QTF was shown in Figure 2. The output laser passed through the QTF gap. Meanwhile, the target gas absorbed laser radiation and generated acoustic wave which was detected by a tiny QTF. Due to its sophisticated piezoelectric effect, the acoustic wave signal can be converted into current signal by QTF. Therefore, the gas concentration can be carried out by detecting the value of piezoelectric current.

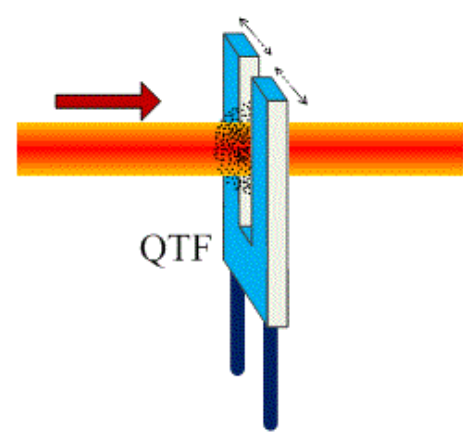

Figure 2: The configuration of detection system with QTF. 
Citation: Ma Y (2017) Trace Gas Sensing Based on Laser Photoacoustic Spectroscopy. Int J Sens Netw Data Commun 5: e111. doi: 10.4172/2090-4886.1000e111

Page 2 of 2

\section{References}

1. Kosterev AA, Bakhirkin YA, Curl RF, Tittel FK (2002) Quartz-enhanced photoacoustic spectroscopy. Opt Lett 27: 1902-1904.

2. Ma Y, Lewicki R, Razeghi M, Tittel FK (2013) QEPAS based ppb-level detection of $\mathrm{CO}$ and $\mathrm{N}_{2} \mathrm{O}$ using a high power CW DFB-QCL. Opt Express 21: 1008-1019.

3. Ma Y, Yu X, Yu G, Li X, Zhang J, et al. (2015) Multi-quartz-enhanced photoacoustic spectroscopy. Appl Phys Lett 107: 021106.
4. Ma Y, He Y, Yu X, Zhang J, Sun R (2016) Compact all-fiber quartzenhanced photoacoustic spectroscopy sensor with a $30.72 \mathrm{kHz}$ quartz tuning fork and spatially resolved trace gas detection. Appl Phys Lett 108: 091115.

5. Ma Y, He Y, Chen C, Yu X, Zhang J, et al. (2016) Planar laser-based QEPAS trace gas sensor. Sensors 16: 989 . 